\title{
Design of a clinician dashboard to facilitate co-decision making in the management of non-specific low back pain
}

\author{
Kerstin Bach ${ }^{1}$. Cindy Marling ${ }^{2}$ (D) . Paul Jarle Mork ${ }^{3}$ - Agnar Aamodt ${ }^{1}$. \\ Frances S. Mair ${ }^{4}$ - Barbara I. Nicholl ${ }^{4}$
}

Received: 13 October 2017 / Revised: 22 October 2018 / Accepted: 24 October 2018 /

Published online: 3 December 2018

(C) The Author(s) 2018

\begin{abstract}
This paper presents the design of a Clinician Dashboard to promote co-decision making between patients and clinicians. Targeted patients are those with non-specific low back pain, a leading cause of discomfort, disability and absence from work throughout the world. Targeted clinicians are those in primary care, including general practitioners, physiotherapists, and chiropractors. Here, the functional specifications for the Clinical Dashboard are delineated, and wireframes illustrating the system interface and flow of control are shown. Representative scenarios are presented to exemplify how the system could be used for codecision making by a patient and clinician. Also included are a discussion of potential barriers to implementation and use in clinical practice and a look ahead to future work. This work has been conducted as part of the Horizon 2020 SELFBACK project, which is funded by the European Commission.
\end{abstract}

Keywords Clinician dashboard - Clinical decision support systems · Co-decision making · Non-specific low back pain · Self-management · Case-based reasoning

\section{Introduction}

SELFBACK is a Horizon 2020 project, involving researchers from four different countries, aiming to solve the challenges related to self-management of non-specific low back pain (Mork and Bach 2018). The Global Burden of Disease Study has identified low back pain as the single leading cause of disability throughout the world. Over half a billion people experienced low back pain in 2016 (Vos et al. 2017). Nearly all low back pain is nonspecific, which means that it can not be attributed to any specific pathological cause, such as fracture, malignancy, or infection (Hartvigsen et al. 2018). The pain itself is a symptom, rather than a disease. It may include muscle ache and shooting or stabbing pain, primarily

Cindy Marling

marling@ohio.edu

Frances S. Mair

Frances.Mair@glasgow.ac.uk

Extended author information available on the last page of the article. 
in the lower back, but sometimes radiating down the leg. It may resolve within a few weeks, in acute cases, or develop into a longer lasting, chronic condition. Non-specific low back pain has a life-time prevalence of 60 to $70 \%$ in industrialized countries, and it affects people of all ages, genders, and cultures (Duthey 2013). The economic burden of non-specific low back pain is high, including the direct costs of medical treatment and the indirect costs of missed work and lost productivity (Hartvigsen et al. 2018).

The goal of the SELFBACK project is to facilitate, improve, and reinforce the selfmanagement of non-specific low back pain. Its aims are to:

1. predict the course of non-specific low back pain, using computer models together with data obtained from wearable fitness trackers and patient questionnaires;

2. generate personalized advice for patients, via a case-based decision support system; and 3. leverage mobile technology to make the system available to patients via a smartphone app.

One novel aspect of the SELFBACK decision support system is the Clinician Dashboard, which facilitates co-decision making. Co-decision making, or shared decision making, has been defined as "an approach where clinicians and patients share the best available evidence when faced with the task of making decisions, and where patients are supported to consider options, to achieve informed preferences" (Elwyn et al. 2010). While patients are responsible for the day-to-day management of their own condition, the Clinician Dashboard allows patients to work together with their clinicians, should they so choose, in order to jointly review data and update self-management plans. Although co-decision making can strengthen the clinician-patient relationship and bring multiple perspectives into consideration when targeting a problem, it is not always readily accepted and adopted in practice (Gravel et al. 2006; Légaré et al. 2010). Therefore, considerable effort has gone into the design of a Clinician Dashboard that has the potential to provide useful decision making information in a manner that will be accepted by both patients and clinicians.

This paper describes the design of the Clinician Dashboard for the SELFBACK project. It begins with a brief overview of the SELFBACK system as a whole, to provide the requisite context. It then continues with the functional specifications for the Clinician Dashboard and provides wireframes to illustrate the system interface. Next, scenarios representing typical system usage by a patient and a clinician are presented. A discussion highlighting challenges and opportunities for using the Clinician Dashboard in clinical practice follows. Finally, the paper ends with a look at future plans and a brief summary and conclusion.

\section{The SELFBACK system}

The SELFBACK system, currently under development, leverages evidence-based pain management guidelines, mobile technologies, and case-based reasoning to tailor patient-specific recommendations for non-specific low back pain and to help patients adhere to these recommendations. In brief, best practice guidelines advocate that the patient is reassured about the good prognosis, stay active, and engage in strength and/or stretching exercises (Airaksinen et al. 2006; van Tulder et al. 2006). Mobile technologies allow patient activity to be monitored, through fitness wristbands, and allow users to interact with the system, through a smartphone app. Case-based reasoning (Bichindaritz and Marling 2006) provides a predictive computer model, based on past cases, that can generate individualized recommendations in accordance with a patient's own goals and progress (Prestmo et al. 2017). 


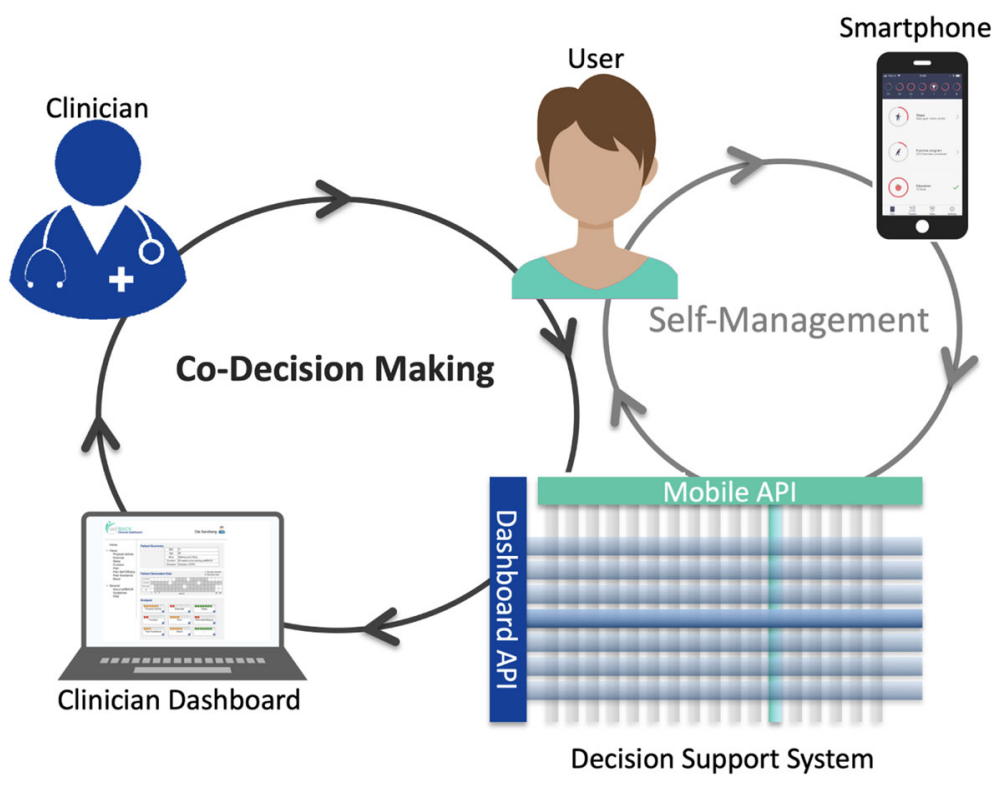

Fig. 1 Overview of the SELFBACK system showing two different application cycles: self-management and co-decision making

The SELFBACK system has two different application cycles, one for self-management and one for co-decision making, as illustrated by Fig. 1.

The patient first encounters SELFBACK when referred by a primary care clinician after presenting with non-specific low back pain. After the referral, the patient will receive an invitation to start the SELFBACK on-boarding process. The patient is asked to fill out a webbased questionnaire that obtains basic data, including demographics, history of low back pain and its treatment, functional ability, and other information needed to assess the patient's status. This data aids in the generation of the initial self-management plan, which evolves as additional data is collected. After the on-boarding is completed, the patient receives access to the mobile app, and the self-management cycle shown in Fig. 1 begins. On a weekly basis, or less frequently once the patient has been using SELFBACK for a while, the app asks follow-up questions about current levels of pain, functional ability, and mood. This allows the self-management plan to be adjusted to account for the progression of the patient's condition. It also presents the patient with educational material, for example, to explain the causes of low back pain or to provide instructions for performing recommended exercises. All data from the app collected prior to and during the self-management cycle is stored and processed by the decision support system (DSS). The DSS analyzes, aggregates and reasons about the provided data. For the self-management component, it tailors exercise, education and physical activity contents using case-based reasoning and heuristics representing stateof-the-art guidelines for low back pain treatment.

While self-management focuses on providing weekly recommendations for each patient, the co-decision making module additionally utilizes data from many patients to visualize the current patient's situation together with similar ones. The focus of this component is to allow a targeted discussion between clinician and patient based on a solid data foundation. Guided by the patient's progress and achievements, similar situations and their prognostics will be provided. 
The self-management component will be built and evaluated prior to the implementation of the co-decision making module, as patient self-management is the primary focus of SELFBACK. However, the Clinician Dashboard, should the patient choose to allow clinician access to their personal data, can extend the SELFBACK system to support co-decisions about the patient's treatment and self-management plan. The Clinician Dashboard targets the interaction of patients with clinicians in primary care, including general practitioners (GPs), physiotherapists, and chiropractors. Its objectives are to:

1. facilitate co-decision making about the treatment, and especially the self-management, of non-specific low back pain;

2. facilitate discussion about barriers and/or expectations for self-management;

3. help the clinician reinforce SELFBACK recommendations for self-management; and

4. provide data to inform the clinician about the effect of the current management approach and to facilitate consideration of other treatment options, including referral to specialized care, if needed.

While the data foundation for both self-management and co-decision making is identical, we implement different Application Programming Interfaces (APIs) to serve the applications. For the co-decision making components, we focus on data aggregations over similar patients as well as the history of self-management data, together with information on pain, function, sleep and quality of life.

\section{Functional specification of the clinician dashboard}

In accordance with the HONcode ${ }^{1}$ principle, the SELFBACK system is not intended to replace the clinician-patient relationship. Rather, it facilitates the clinician-patient relationship by enabling the clinician to work jointly with the patient to review progress and develop effective personalized self-management plans. We developed the concept of the Clinician Dashboard in collaboration with primary care researchers with different clinical backgrounds and broad experience in physiotherapy and general practice. Our expert group met regularly to discuss the data foundation provided by the SELFBACK system and possible scenarios for using it in clinical practice to enrich discussions with patients. Once the overall user scenario was set, we started paper prototyping, which eventually led to the screens we present in this paper. The overall flow of the system as well as the content of each screen was discussed in the context of user scenarios, where we focused on selecting and emphasizing information that should be presented to the patient.

In our vision, the clinician applying the SELFBACK approach is equipped with a web-based Clinician Dashboard to be used for co-decision making with the patient. The SELFBACK server is accessed via a secure connection through the web interface. It will typically be run on a PC and can therefore display more comprehensive information than the patient's smartphone app. The web interface will display statistics on (1) the progression of symptoms (e.g., pain, function), (2) physical activity level, and (3) adherence to exercise plans, for the consulting patient and for similar patients using SELFBACK.

The use of SELFBACK is initiated when a clinician (a GP, physiotherapist, or chiropractor) refers a patient with low back pain to the system. This referral associates the patient with the practice and limits access to the patient's data to clinicians in the practice. The

\footnotetext{
${ }^{1}$ http://www.hon.ch/HONcode/Patients/method.html
} 
clinician logs into the system with a username and password. The clinician only has access to detailed data for patients in his or her own practice who consent to data sharing. To maintain the privacy of patient data, a unique patient ID is generated for each patient every week and provided to the patient via the SELFBACK smartphone app. During a consultation, the patient shares the current weekly ID with the clinician to enable data access to all of the patient's data, including all historic data, for the duration of that week, via the Clinician Dashboard. Once a new weekly ID is generated, the clinician can no longer access the patient's data, until and unless the patient shares the new ID during a subsequent consultation. The timing of consultations will be dependent on the particular patient's needs and the clinician's practice. The patient may choose to share their ID with more than one clinician, e.g., their GP and their physiotherapist.

The Clinician Dashboard uses the same data sources as the SELFBACK smartphone app, but it aggregates and prepares the data differently in order to display historical data for an individual patient and for similar patients or groups. The included patient information centers on measures of:

- Physical Activity (i.e., activity vs. inactivity)

- Exercise

- Sleep Quality

- Functional Ability

- Pain Level

- Pain Self-Efficacy (i.e., belief in the ability to carry out daily activities despite the pain)

- Fear Avoidance (i.e., fear of pain as it deters adherence to exercise plans)

- Mood

For co-decision making, the clinician logs into the system, enters the ID provided by the patient, and then accesses data for that individual patient during their appointment. This enables the clinician and the patient to review and discuss the data together. Because appointment time is limited, the dashboard has a simple and intuitive interface that quickly focuses users on problems that warrant mutual discussion. An initial screen graphically displays the patient's progress with respect to physical activity, exercise, sleep, function, pain level, pain self-efficacy, fear avoidance, and mood. This helps to clarify, at a glance, which aspects of self-management are progressing well and which are not. Users can then choose to click on only those aspects that are not progressing well for additional detail that may help to inform co-decision making. Upon clicking, detailed data for the patient is displayed over time. For some items, where an external reference could be helpful, e.g., physical activity, there is an additional plot of the patient's progress in comparison to that of a reference group of similar patients. Furthermore, each plot is printable, so that the clinician can offer the patient a printout to take home. The printout will contain only the annotated plot, without additional patient information.

\section{Wireframes}

In this section, wireframes are presented to illustrate the overall flow of control of the Clinician Dashboard. Figure 2 shows the flow through the Clinician Dashboard during a co-decision making session between a clinician and a patient. First, the clinician logs in, by entering a username and password. This enables access to data for the patients in the clinician's practice. To view the dashboard for a specific patient, the clinician next enters the 


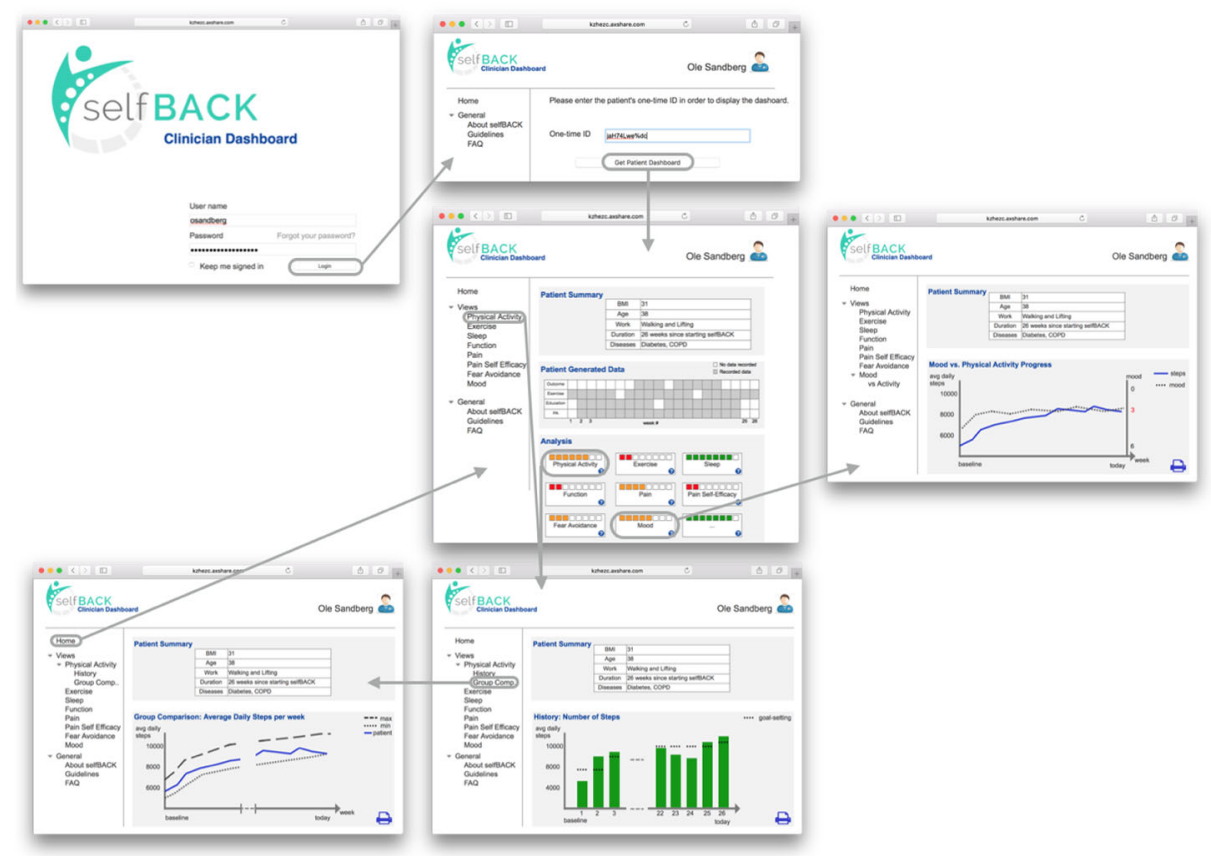

Fig. 2 Overview of the flow through the Clinician Dashboard

patient's ID. The patient finds this ID in the SELFBACK smartphone app and provides it to the clinician for entry.

Once a valid patient ID for the clinician's practice is entered, the main page of the Patient View opens, as shown in Fig. 3. As can be seen in the figure, this page contains the following components:

- the Header, displaying the name of the clinician

- a Navigation Bar, on the left, providing access to all system sub-views

- a Patient Summary, displaying patient specific information, which may be used to compare the patient to other similar patients

- an overview of Patient Generated Data, indicating how frequently the patient has provided data to the system

- an Analysis section, containing nine tiles, one for each co-decision making topic or aspect

The tiles in the Analysis section indicate, at a glance, how well the patient has progressed for each aspect. A bar at the top of each tile displays green (6-8 filled blocks) for good progress, yellow (3-5 filled blocks) for potential challenges, or red (1-2 filled blocks) to indicate the need for an intervention. Two visual indicators, color and number of filled blocks, are used together to clearly illustrate progress. To start a discussion with a patient about a particular topic, the clinician clicks on the tile to display the next screen.

Once a tile has been selected, more detailed information is displayed. Consider, for example, that the clinician has clicked on the Physical Activity tile. This brings up a history view, showing to which degree the patient has achieved their physical activity goals, as shown in 


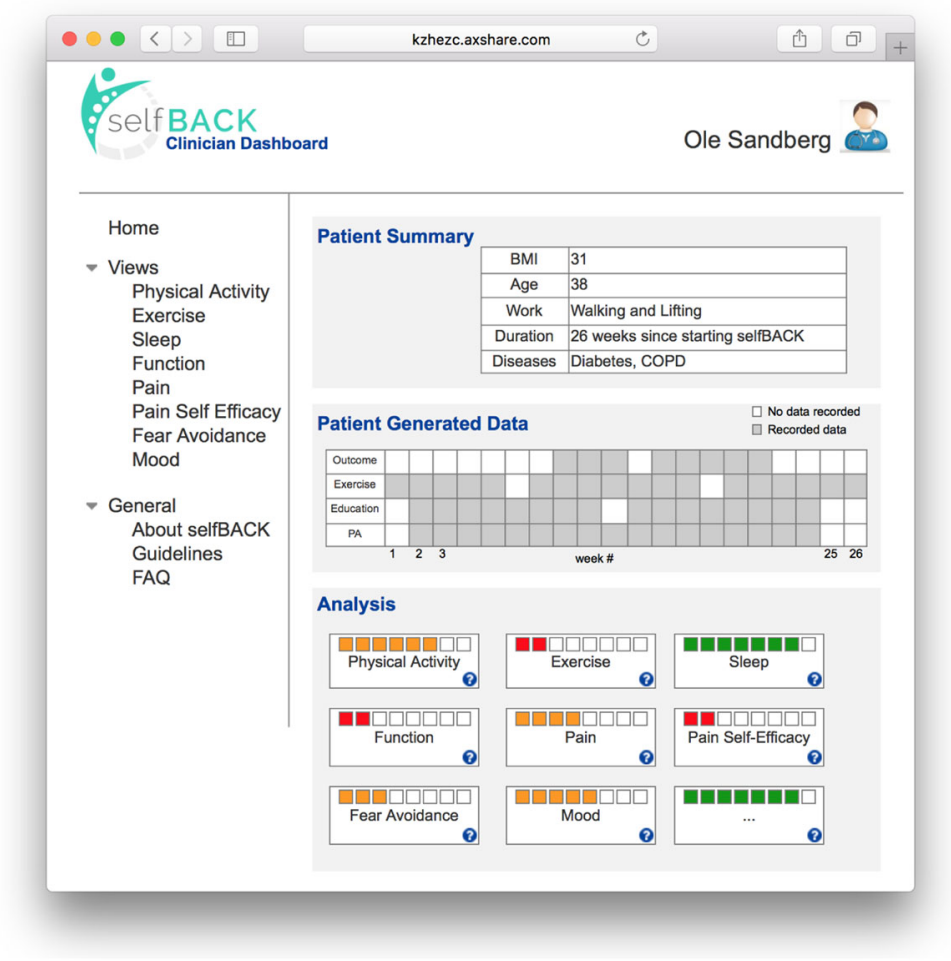

Fig. 3 The main page of the Patient View

Fig. 4. This screen shows how many steps the patient could walk each day when they began using SELFBACK (the baseline), plus the number of daily steps walked for the first three weeks and the most recent few weeks of the program. The clinician can click on the dashed line between these weeks to fill in data for the middle weeks, if desired. The clinician can also choose to use the navigation bar on the left to move to another aspect of co-decision making. For example, the clinician may choose to show the patient how their progress compares to that of other similar patients, by clicking on Group Comparison in the navigation bar.

Figure 5 shows an example of a Group Comparison for Physical Activity. For the Group Comparison view, information about patients who are similar to the current patient is retrieved from the SELFBACK case base. Minimum and maximum achievements for this reference group are used to show how the achievements of the current patient compare to those of similar patients. Only patients with positive outcomes, such as decreased pain, improved pain self efficacy, and better mood, are included in this reference group. This provides a framework for positing that, if the patient's achievement lies between the minimum and maximum, a positive outcome is foreseen. If the patient's achievement is above the maximum for his or her group, this may provide positive feedback for the patient, unless other aspects (e.g., pain) indicate that the patient could be overdoing it and should try to adhere to the limits of their self-management plan. Similarly, if the patient's achievement is below the minimum, the clinician can also address that issue. 


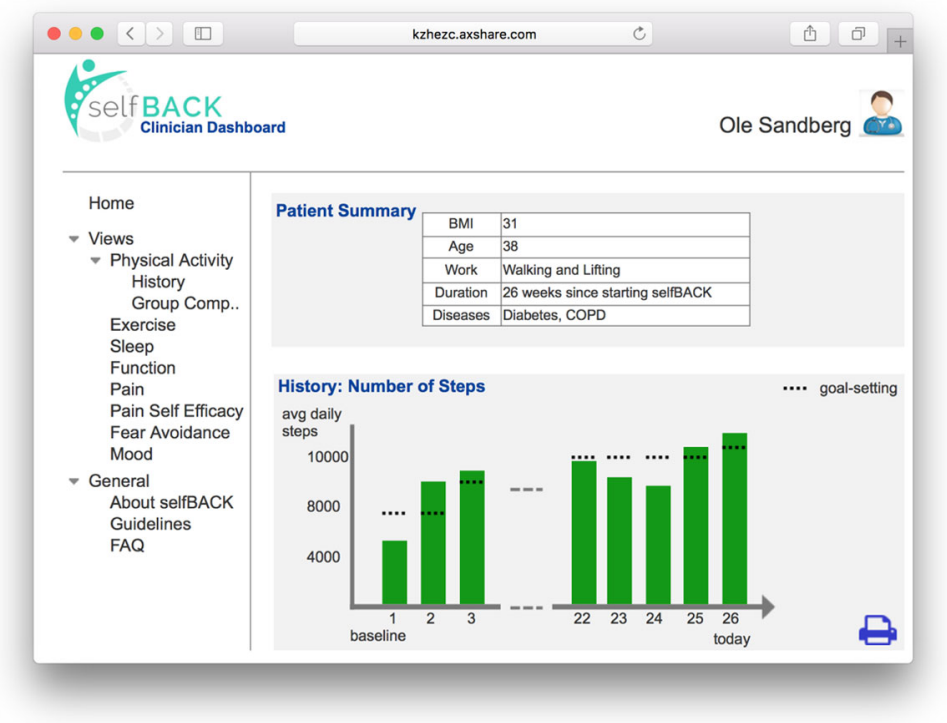

Fig. 4 The history view for the Physical Activity example

Should clinicians want to review established international clinical guidelines for managing non-specific low back pain, they can do so within the Clinician Dashboard. However, because these documents are lengthy, and patient appointments are time-limited, summaries are provided for each co-decision making topic. Each of the tiles in the Analysis section of

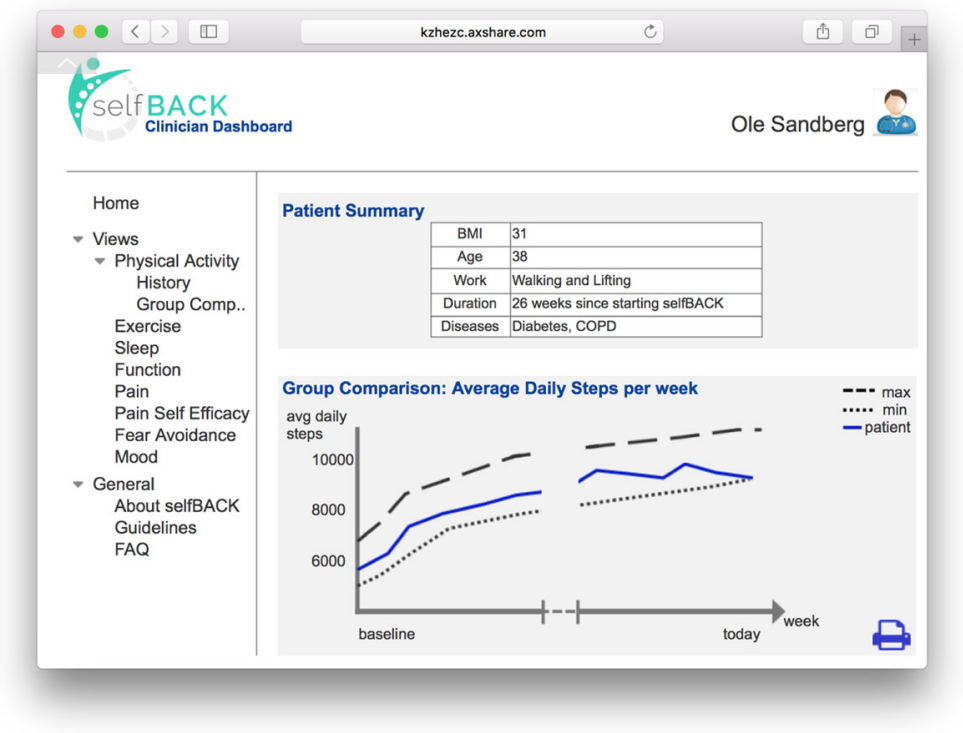

Fig. 5 The group comparison view for the Physical Activity example 
the main page of the Patient View has a question mark in its lower right hand corner. Hovering over a question mark with the cursor will bring up a summary recommendation based on internationally recognized and/or institutionally mandated clinical guidelines. Examples from Exercise, Function, and Mood follow:

\section{Exercise}

- Take people's preferences and capabilities into account when choosing type of exercise

- Choose exercises that target relevant muscle groups

\section{Function}

- Encourage a patient with low back pain to continue with normal activities, including returning to work or normal activities of daily living

\section{Mood}

- Consider cognitive behavioral approaches, in combination with other self-management approaches

- Encourage a combined physical and psychological program

\section{Illustrative usage scenarios}

The following scenarios illustrate how the Clinician Dashboard could be used in everyday practice, from the vantage points of a patient and a clinician.

\subsection{Patient}

A female patient (age 38 years, body mass index $31 \mathrm{~kg} / \mathrm{m}^{2}$, occupation cleaner) was advised by her GP to start using SELFBACK to help her manage her low back pain. She has had her low back pain for almost six months, and it does not seem to be getting any better. She has always found that walking makes her low back pain worse, and she believes that she gets enough physical activity from her job as a cleaner. She reports using over-thecounter pain killers to enable her to do her job. She reports low quality of life, as she is unable to enjoy activities with friends and family. She has been wearing the activity tracking wristband and using the SELFBACK smartphone app for one month. She says that she has been following the system's advice, but that she has not been able to achieve the steps goals, because walking aggravates her pain. She wants to discuss her low back pain with her GP.

The GP is able to bring up her SELFBACK profile on the computer after she gives him her ID from the smartphone app, and they can look at the computer screen together. The patient finds this useful and can see that she is gradually increasing the number of steps that she is taking, getting closer to her goal. The GP starts to talk to her about what might be stopping her from doing more exercise, and he shows her some graphs on the screen. Her belief that exercise will result in pain has remained constant over the past month. Her GP suggests that she watch some of the educational videos on the SELFBACK smartphone app again to learn more about the causes of low back pain and to see if that might help her try to walk more. The GP suggests to the patient that the exercise would be helpful not only for her low back pain but for her weight and mood as well. The GP shows the patient a screen where she can see her pain score compared to those of other people like her. She can see that, if she keeps 
using the smartphone app, it looks as though her low back pain will improve. The patient takes this as encouragement but is also confused about how the GP can be sure about this. The GP explains that this information is based on other patients who are similar to her in terms of age, sex, and other important characteristics, such as her weight. The patient says that she will try to do this and will come back and see the GP again in about two months' time.

\subsection{Clinician}

A GP has been recommending the use of SELFBACK to a number of patients, some with new episodes of acute low back pain and others who have had low back pain for a long time or who have had recent flare-ups in their pain. The above patient has been coming to see the GP regularly (every one to two weeks) for her low back pain for six months. One month ago, he suggested that she start to use SELFBACK. When the patient comes into the consulting room, the GP asks her how she is and how her low back pain is. She reports that she has been using the SELFBACK smartphone app, but that her pain has not gotten any better. The GP asks the patient if he can look at her SELFBACK profile with her. The patient provides the GP with a personal ID code, and the GP enters this code into the system and brings up the patient's profile. The GP can see from the pain intensity progress graph that her pain level has actually decreased from 7, when she first started using SELFBACK, to 5, just two days ago. Showing this graph to the patient allowed the GP to highlight this progress. As this patient has a physically active job but does not think that exercise helps her, the GP asks about her exercise and walking routines. The GP brings up the activity progress screen and sees that, although the patient is not reaching her target number of steps, she has increased her activity from the first week. The GP shows her a plot of her activity in relation to other people like her, of similar weight and age, and she can see that she could be more active. The GP asks her why she is not doing more, and she reiterates her worry that it will cause more pain. Indeed, the GP can see this when they look at the fear avoidance graph; this score has not changed from her first use of SELFBACK. They discuss why this might be, and the GP is able to reassure the patient. He encourages her to pay special attention to the educational component of the smartphone app to learn more about what causes low back pain, so that she can see that physical activity is not damaging. The patient leaves the consultation with the GP feeling positive, as she can see that other similar patients who have followed this guidance have had an improvement in their pain. The GP suggests that she wait for two months before coming back, unless there is something different or changed about her low back pain.

\section{Potential barriers to system usage}

Despite the focus on and interest in the use of computerized assistance and shared decision making, implementation in clinical practice has proved difficult (Berner 2009; Coiera et al. 2009), and several challenges remain to be solved. Studies have identified the following barriers to introducing decision support systems based on patient generated data into clinical practice (Chiauzzi et al. 2015; Cohen et al. 2016; Elwyn et al. 2010; McGee-Lennon et al. 2017):

1. technical/ethical issues (collection and storage of patient generated data, patient privacy concerns); 
2. time constraints and usability of patient generated data in clinical practice (access to the data during a consultation, how to use and interpret the data);

3. cultural issues within the clinical community (lack of practice workflows and protocols related to the collection and use of patient generated data); and

4. lack of confidence in system reliability by health care professionals.

The main barriers identified as potentially impacting the implementation and use of the Clinician Dashboard are clinician time constraints and patient data privacy concerns.

A GP typically has only ten minutes available per patient visit. This is why the Clinician Dashboard was designed to quickly highlight problems via indicator bars on the tiles of the main page of the Patient View. It is also why summaries of clinical guidelines for managing highlighted problems are included with the hover of a mouse on each tile. The system was designed to minimize the number of clicks needed to find data that is applicable to the co-decision making session in progress. The navigation bar ensures that the clinician can quickly move from any co-decision making topic to the next.

Patient data privacy concerns take precedence over speed of use in the initial two screens, where the clinician must provide valid login credentials and a patient ID for data access. It is still technically challenging to ensure that patient data can be leveraged for legitimate health care purposes, while preventing its unauthorized access or misuse. To enable wellinformed decisions, it is important to have access to valid and relevant data for the consulting patient. This includes patient generated data, or data collected by the patient him or herself. For conditions where self-management constitutes a core component, like low back pain, it would be particularly relevant to have time-series data about the self-management process at regular follow-up consultations. In the SELFBACK system, such time-series data includes repeated achievement (self-management) and outcome (symptom progression) measurements. When clinicians have access to patient generated data in a structured setting, they report three main benefits with direct relevance for shared decision making (Cohen et al. 2016):

1. improved insight into the condition of individual patients;

2. improved general and clinical information about the patient; and

3. better insight into symptom variation and health behavior between consultations, enabling revision of treatment plans to improve outcomes and avoid unnecessary consultations.

Another potentially important input to the shared decision making process is data collected over time by wearable activity tracking devices. Such data may be of particular relevance for follow-up of patients with chronic conditions and diseases where self-management is a key component of disease management. Insights into the progression and impact of symptoms, based on wearable activity monitors, have already been reported (Shammas et al. 2014). Data from activity tracking wristbands will be collected for SELFBACK, and could potentially be used to enhance the co-decision making process.

As to the patient perspective, surveys have shown that a majority of patients are willing to share their health data with their own physicians, and more broadly for purposes of research or to help other patients like themselves. However, these surveys also show that most consumers have privacy concerns about data sharing and are aware of potential negative consequences (Chiauzzi et al. 2015). SELFBACK's use of weekly expiring identifiers is just one means of protecting patient privacy. The implementation of robust protocols to ensure patient privacy remains as future work. 


\section{Related work}

It has been over 50 years since the first clinical decision support systems (CDSSs) were implemented. Historically, these systems were built to assist clinicians with various tasks, including diagnosis, therapy planning and critiquing, drug prescription, information retrieval, and image interpretation. They have provided reminders of preventive measures (e.g., prescribing antibiotics before surgery) and alerts about potential dangers (e.g., adverse drug interactions) (Coiera et al. 2009). Traditionally, it has been assumed that the human decision maker using the CDSS is a physician, although sometimes, other clinicians, such as nurses, are supported as well. Clinician resistance to the use of CDSSs has been a longstanding problem, with technical, institutional and cultural barriers to system adoption (Berner 2009).

The use of CDSSs by patients is a relatively recent development. Over the past decades, health care systems and politicians have placed increasing importance on the relationship between patient choice and clinical decision making (Coiera et al. 2009). With shared decision making, patients are encouraged to assess the benefits and risks of different available treatment or management options. This is intended to increase patient engagement and ensure that patient autonomy is respected. To facilitate shared decision making, the patient and clinician can utilize different decision aids, e.g., customized software that can aggregate current best evidence and inform the process. IBM's Watson is a well-known recent example of such computerized assistance for clinical decision making (Lee 2014).

At the beginning of the SELFBACK project, we undertook a systematic review of digital support interventions for the self-management of low back pain (Nicholl et al. 2017). Included in this review were interactive interventions delivered via computers, mobile phones, and/or hand-held devices. Nine studies were identified, including six completed randomized controlled trials (RCTs) and three descriptions of protocols for future RCTs. The evidence to date for the efficacy of such interventions was found to be weak. The patient population in the RCTs was skewed toward white, middle-aged, well-educated females. There was no report of the economic cost-effectiveness of any intervention. Only one of the studies reported that the intervention had a positive effect on their primary outcome (Carpenter et al. 2012).

These prior support systems differed considerably from SELFBACK. For one thing, unlike SELFBACK, these systems did not include any machine learning component to tailor treatment to the individual patient. Rather, they relied upon established guidelines and best practices to provide tailored support. Furthermore, none of the systems included support for co-decision making, as facilitated by SELFBACK's Clinician Dashboard. The patient was the sole user of all of the support interventions except one. The exception simply included a web-based discussion forum, in which the patient, peers, and health care professionals could participate (Krein et al. 2010, 2013).

Machine learning models were recently evaluated and adopted to support self-referral decisions for patients with low back pain (Nijeweme-d'Hollosy et al. 2016, 2018). The machine learning approaches considered were decision trees, random forests, and boosted trees. The task at hand was enabling a person with acute low back pain to determine whether to consult a general practitioner, consult a physiotherapist, or engage in self-care. The goal is to prevent acute pain from progressing to chronic pain by obtaining the right type of care at the right time. In machine learning terms, this is a classification system which places the patient into one of three categories: GP, physiotherapist, or self-care. It does not provide suggestions for managing the condition beyond this classification. 
A systematic review of smartphone apps for the self-management of low back pain has also been published (Machado et al. 2017). This review included 61 apps for both iPhones and Android smart phones. No RCTs for these apps were reported in the scientific literature. While nearly all apps recommended interventions in line with clinical practice guidelines, the authors found apps, in general, to offer low quality information and to be of questionable credibility. The authors conclude that the proliferation of smarthphone apps without adequate regulation or assessment raises concerns as to their efficacy and makes it difficult for patients and care providers to select high quality apps.

A short-term retrospective study has been reported which evaluates the efficacy of the Kaia app (Huber et al. 2017). Kaia, which is available for iPhones and Androids, uses a multidisciplinary approach, including education about back pain, physiotherapy, and mindfulness techniques. In a 12-week review of the data for 180 patients who used the app, there was a significant and clinically relevant reduction in self-reported pain levels. The authors, who plan a prospective RCT for the future, believe that this is one of the first empirical assessments of a low back pain management smartphone app.

While, to the best of our knowledge, SELFBACK is the only low back pain decision support system to incorporate co-decision making, co-decision making has been used in decision support systems for other medical conditions. An early example was PANDEX, which supports shared decision making for genetic consultation surrounding prenatal tests (Segal and Shahar 2009). PANDEX uses a Bayesian probabalistic model to calculate the expected utilities of possible courses of action. There is an emphasis on explaining and exploring options, so that patients can make truly informed decisions in a domain where different individuals will have different moral and ethical considerations as well as varying perspectives on the desirability of different outcomes. Co-decision making is also key to a system for the personal health optimization of people with bipolar disorder (Eiring et al. 2017). Bipolar disorder is a chronic condition plagued by suboptimal health outcomes, exacerbated by poor adherence to medication regimens. Medicine optimization for this condition combines the best available evidence with patient values and preferences, using shared decision making. The authors posit that the limited efficacy of prior clinical decision support aids is due, in part, to their not allowing patients and clinicians to jointly interact with the system. This system supports patients, clinicians and caregivers in the shared decision making process. Another clinical decision support tool incorporating co-decision making is iPrevent, for assessing and managing breast cancer risk (Collins et al. 2016). iPrevent estimates a woman's personal breast cancer risk using validated risk assessment models and facilitates discussions between women and their health care providers about evidence-based measures to manage that risk. As in SELFBACK's Clinician Dashboard, information is displayed using easily comprehensible graphical formats, and users have options they can click on to review additional details.

\section{Future work}

The design of the Clinician Dashboard has proceeded in parallel with the implementation of the first four modules of the SELFBACK system. The SELFBACK system, except for the Clinician Dashboard, is scheduled for evaluation via a randomized controlled trial in mid-2018 through mid-2020. A total of 305 patients with non-specific low back pain, in Norway, Denmark, and the United Kingdom, will participate in the trial. Each patient will either use SELFBACK, or serve as a control, for a period of nine months. Although the Clinician Dashboard will not be included in this randomized controlled trial, it is possible 
that the trial will illuminate needed system changes that could impact the implementation of the Clinician Dashboard. The next steps for the Clinician Dashboard are to:

1. review the wireframes with clinical practitioners and patient groups to obtain feedback from prospective users;

2. create a demonstrator connected to SELFBACK for display of patient data;

3. elicit clinician buy-in and participation; and

4. implement and test necessary data privacy and security measures.

\section{Summary and conclusion}

This paper has presented the design of a Clinician Dashboard to facilitate co-decision making between patients and clinicians. The Clinician Dashboard is intended to extend the SELFBACK system, which supports the self-management of non-specific low back pain. SELFBACK is a European Union project involving researchers from four different countries. The system as a whole, with the exception of the Clinician Dashboard, is scheduled for evaluation via a randomized controlled trial beginning in 2018. In this paper, the functional specifications for the Clinician Dashboard are delineated, and wireframes portraying the system interface and flow of control are shown. Scenarios illustrating representative system usage, from the vantage points of a patient and a clinician, are provided. Potential barriers to system implementation and use in clinical practice are discussed. Of primary importance are minimizing the time it takes the clinician to use the system during a typical consultation and protecting the privacy of the patient's personal health data. The design of the Clinician Dashboard aims to address these potential barriers prior to implementation, to ensure that the system will be acceptable to both patients and clinicians.

Co-decision making has the potential to strengthen the clinician-patient relationship, increase patient engagement, ensure that patient autonomy is respected, improve clinician insight into individual patient problems, and support evidence-based treatment planning. Most importantly for SELFBACK, it may lead to improved self-management plans and better adherence to self-management, with a concomitant reduction in the number of professional consultations required. The design of the Clinician Dashboard, as described in this paper, will be implemented in future work. We envision that a similar design approach could be fruitfully applied to the development of other self-management tools, potentially broadening the impact of this work beyond the SELFBACK use case.

Acknowledgements This work has been conducted as part of the SELFBACK project, which has received funding from the European Union's Horizon 2020 research and innovation programme under grant agreement No 689043.

Open Access This article is distributed under the terms of the Creative Commons Attribution 4.0 International License (http://creativecommons.org/licenses/by/4.0/), which permits unrestricted use, distribution, and reproduction in any medium, provided you give appropriate credit to the original author(s) and the source, provide a link to the Creative Commons license, and indicate if changes were made.

\section{References}

Airaksinen, O., Brox, J., Cedraschi, C., Hildebrandt, J., Klaber-Moffett, J., Kovacs, F., Mannion, A., Reis, S., Staal, J., Ursin, H., et al. (2006). Chapter 4. European guidelines for the management of chronic nonspecific low back pain. European Spine Journal, 15, s192-s300. 
Berner, E.S. (2009). Clinical decision support systems: state of the art. AHRQ publication no 09-0069-EF.

Bichindaritz, I., \& Marling, C. (2006). Case-based reasoning in the health sciences: What's next? Artificial Intelligence in Medicine, 36(2), 127-135.

Carpenter, K.M., Stoner, S.A., Mundt, J.M., Stoelb, B. (2012). An online self-help CBT intervention for chronic lower back pain. The Clinical Journal of Pain, 28(1), 14.

Chiauzzi, E., Rodarte, C., DasMahapatra, P. (2015). Patient-centered activity monitoring in the selfmanagement of chronic health conditions. BMC Medicine, 13(1), 77.

Cohen, D.J., Keller, S.R., Hayes, G.R., Dorr, D.A., Ash, J.S., Sittig, D.F. (2016). Integrating patientgenerated health data into clinical care settings or clinical decision-making: lessons learned from Project HealthDesign. JMIR Human Factors, 3(2), e26.

Coiera, E., Lau, A., Tsafnat, G., Sintchenko, V., Magrabi, F. (2009). The changing nature of clinical decision support systems: a focus on consumers, genomics, public health and decision safety. Yearbook of Medical Informatics, 1, 84-95.

Collins, I.M., Bickerstaffe, A., Ranaweera, T., Maddumarachchi, S., Keogh, L., Emery, J., Mann, G.B., Butow, P., Weideman, P., Steel, E., et al. (2016). iprevent $囚$ : a tailored, web-based, decision support tool for breast cancer risk assessment and management. Breast Cancer Research and Treatment, 156(1), 171-182.

Duthey, B. (2013). Background paper 6.24. low back pain. World Health Organisation (WHO)(ed) Priority medicines for Europe and the world 'A public health approach to innovation' Geneva: WHO.

Eiring, Ø., Nytrøen, K., Kienlin, S., Khodambashi, S., Nylenna, M. (2017). The development and feasibility of a personal health-optimization system for people with bipolar disorder. BMC Medical Informatics and Decision Making, 17(1), 102.

Elwyn, G., Laitner, S., Coulter, A., Walker, E., Watson, P., Thomson, R. (2010). Implementing shared decision making in the NHS. BMJ, 341, 971-973.

Gravel, K., Légaré, F., Graham, I.D. (2006). Barriers and facilitators to implementing shared decision-making in clinical practice: a systematic review of health professionals' perceptions. Implementation Science, $1(1), 16$.

Hartvigsen, J., Hancock, M.J., Kongsted, A., Louw, Q., Ferreira, M.L., Genevay, S., Hoy, D., Karppinen, J., Pransky, G., Sieper, J., et al. (2018). What low back pain is and why we need to pay attention. The Lancet, 391, 2356-2367.

Huber, S., Priebe, J.A., Baumann, K.M., Plidschun, A., Schiessl, C., Tölle, T. R. (2017). Treatment of low back pain with a digital multidisciplinary pain treatment app: short-term results. JMIR Rehabilitation and Assistive Technologies, 4(2), e11.

Krein, S.L., Metreger, T., Kadri, R., Hughes, M., Kerr, E.A., Piette, J.D., Kim, H.M., Richardson, C.R. (2010). Veterans walk to beat back pain: study rationale, design and protocol of a randomized trial of a pedometer-based internet mediated intervention for patients with chronic low back pain. $B M C$ Musculoskeletal Disorders, 11(1), 205.

Krein, S.L., Kadri, R., Hughes, M., Kerr, E.A., Piette, J.D., Holleman, R., Kim, H.M., Richardson, C.R. (2013). Pedometer-based internet-mediated intervention for adults with chronic low back pain: randomized controlled trial. Journal of Medical Internet Research, 15(8), e181.

Lee, H. (2014). Paging Dr. Watson: IBM's Watson supercomputer now being used in healthcare. Journal of AHIMA, 85(5), 44-47.

Légaré, F., Ratté, S., Stacey, D., Kryworuchko, J., Gravel, K., Graham, I.D., Turcotte, S. (2010). Interventions for improving the adoption of shared decision making by healthcare professionals. Cochrane Database of Systematic Reviews, 5(5).

Machado, G.C., Pinheiro, M.B., Lee, H., Ahmed, O.H., Hendrick, P., Williams, C., Kamper, S.J. (2017). Smartphone apps for the self-management of low back pain: A systematic review. Best Practice \& Research Clinical Rheumatology.

McGee-Lennon, R.M., Bouamrane, M.M., Devlin, M.A., O’Connor, S., O'Donnell, C., Chetty, U., Agbakoba, R., Bikker, A., Grieve, E., Finch, T., Watson, N., Wyke, S., Mair, S.F. (2017). Readiness for delivering digital health at scale: Lessons from a longitudinal qualitative evaluation of a national digital health innovation program in the United Kingdom. J Med Internet Res, 19(2), e42.

Mork, P.J., \& Bach, K. (2018). A decision support system to enhance self-management of low back pain: Protocol for the selfBACK project. JMIR Research Protocols, 7(7), e167.

Nicholl, B.I., Sandal, L.F., Stochkendahl, M.J., McCallum, M., Suresh, N., Vasseljen, O., Hartvigsen, J., Mork, P.J., Kjaer, P., Søgaard, K., et al. (2017). Digital support interventions for the self-management of low back pain: a systematic review. Journal of Medical Internet Research, 19(5), e179.

Nijeweme-d'Hollosy, W.O., van Velsen, L.S., Soer, R., Hermens, H.J. (2016). Design of a web-based clinical decision support system for guiding patients with low back pain to the best next step in primary healthcare. In: Proceedings of the 9th international joint conference on biomedical engineering systems 
and technologies (BIOSTEC 2016), SCITEPRESS-Science and Technology Publications, vol. 5, pp. 229-239.

Nijeweme-d'Hollosy, W.O., van Velsen, L., Poel, M., Groothuis-Oudshoorn, C.G., Soer, R., Hermens, H. (2018). Evaluation of three machine learning models for self-referral decision support on low back pain in primary care. International Journal of Medical Informatics, 110, 31-41.

Prestmo, T., Bach, K., Aamodt, A., Mork, P.J. (2017). Evolutionary inspired adaptation of exercise plans for increasing solution variety. In Aha, D., \& Lieber, J. (Eds.) Case-based reasoning research and development, Proceedings of the 25th international conference (ICCBR-17) (pp. 272-286): Springer.

Segal, I., \& Shahar, Y. (2009). A distributed system for support and explanation of shared decision-making in the prenatal testing domain. Journal of Biomedical Informatics, 42(2), 272-286.

Shammas, L., Zentek, T., von Haaren, B., Schlesinger, S., Hey, S., Rashid, A. (2014). Home-based system for physical activity monitoring in patients with multiple sclerosis (pilot study). Biomedical Engineering Online, 13(1), 10.

van Tulder, M., Becker, A., Bekkering, T., Breen, A., Gil del Real, M.T., Hutchinson, A., Koes, B., Laerum, E., Malmivaara, A. (2006). Chapter 3. European guidelines for the management of acute nonspecific low back pain in primary care. European Spine Journal, 15, s169-s191.

Vos, T., Abajobir, A.A., Abate, K.H., Abbafati, C., Abbas, K.M., Abd-Allah, F., Abdulkader, R.S., Abdulle, A.M., Abebo, T.A., Abera, S.F., et al. (2017). Global, regional, and national incidence, prevalence, and years lived with disability for 328 diseases and injuries for 195 countries, 1990-2016: a systematic analysis for the global burden of disease study 2016. The Lancet, 390(10100), 1211-1259.

\title{
Affiliations
}

\section{Kerstin Bach ${ }^{1}$ - Cindy Marling ${ }^{2}$ (D) . Paul Jarle Mork ${ }^{3}$ - Agnar Aamodt ${ }^{1}$. Frances S. Mair ${ }^{4}$ Barbara I. Nicholl ${ }^{4}$}

\author{
Kerstin Bach \\ kerstin.bach@ntnu.no \\ Paul Jarle Mork \\ paul.mork@ntnu.no \\ Agnar Aamodt \\ agnar.aamodt@ntnu.no \\ Barbara I. Nicholl \\ Barbara.Nicholl@glasgow.ac.uk
}

1 Department of Computer Science, Norwegian University of Science and Technology (NTNU), Sem Sælandsvei 7-9, NO-7491 Trondheim, Norway

2 School of Electrical Engineering and Computer Science, Ohio University, Athens, OH 45701, USA

3 Department of Public Health and Nursing, Norwegian University of Science and Technology (NTNU), Håkon Jarls gate 11, NO-7491, Trondheim, Norway

4 Institute of Health and Wellbeing, University of Glasgow, 1 Horselethill Road, Glasgow G12 9LX, UK 